\title{
Endoscopic augmentation of gastroesophageal junction using a full-thickness endoscopic suturing device
}

\section{(ㄷ)(1) $\odot$}

Authors

Jimin Han', Matthew Chin ${ }^{2}$, Kyle J. Fortinsky², Reem Sharaiha ${ }^{3}$, Christopher J. Gostout ${ }^{4}$, Kenneth J. Chang ${ }^{2}$

Institutions

1 Department of Internal Medicine, Catholic University of Daegu School of Medicine Daegu, South Korea

2 H. H. Chao Comprehensive Digestive Disease Center, University of California Irvine Medical Center, Orange, California, USA

3 Weill Cornell Medicine, Division of Gastroenterology and Hepatology, Department of Medicine New York, New York, USA

4 Division of Gastroenterology and Hepatology, Mayo Clinic College of Medicine, Rochester, Minnesota, USA

submitted 8.2.2018

accepted after revision 3.4 .2018

\section{Bibliography}

DOI https://doi.org/10.1055/a-0603-3693 |

Endoscopy International Open 2018; 06: E1120-E1125

(c) Georg Thieme Verlag KG Stuttgart · New York

ISSN 2364-3722

Corresponding author

Kenneth J. Chang, Department of Gastroenterology, University of California Irvine Medical Center, 101 The City Dr. Blvd., Orange, CA 92686

kchang@uci.edu

\section{ABSTRACT}

Background and study aims This preliminary study was conducted to determine the feasibility and safety of endoscopic augmentation of the gastroesophageal junction (GEJ) using the Apollo OverStitch endoscopic suturing system in patients with gastroesophageal reflux disease (GERD) symptoms.

Patients and methods Endoscopic augmentation of GEJ was performed on 10 consecutive patients and the data were analyzed retrospectively. Using a double-channel gastroscope affixed to the endoscopic suturing platform, interrupted sutures were placed on the gastric side of the GEJ in 2 layers in order to create a narrowed and elongated GEJ.

Results Technical success was achieved in all patients, including those with a history of previous antireflux procedures $(n=7)$ and those with a hiatal hernia $(n=6)$. The median follow-up duration was 5 mo (range: 2 -12). The median pre-procedure GERD-Health Related Quality of Life Questionnaire improved from 20 (range: $11-45)$ to a postprocedure score of 6 (range: $3-25)(P=0.001)$. The median duration of GERD symptom improvement after the procedure was 1 mo (range: $0.5-4$ ). Adverse events were limited to 1 patient who developed nausea and vomiting, which was self-limited.

Conclusions The use of a novel endoscopic suturing technique for the treatment of GERD is feasible and safe. The procedure resulted in short-term GERD symptom improvement. Further prospective studies using refined techniques are currently underway to improve durability and to prove efficacy.

\section{Introduction}

Gastroesophageal reflux disease (GERD) is one of the most common chronic conditions, with an estimated prevalence as high as $27.8 \%$ in North America [1] and more than 9 million patient visits per year [2]. While proton pump inhibitors (PPIs) are the mainstay of therapy for GERD, up to $45 \%$ of patients treated with PPIs report persistent symptoms [3], including regurgitation or extraesophageal manifestations [3]. While PPIs are widely used and relatively safe, there are some risks of being on long-term PPI, including chronic kidney disease, hypomag- nesemia, hip fractures, community acquired pneumonias, and Clostridium difficile infection [4].

In PPI-refractory or PPI-adverse patients, endoscopic or surgical alternatives may be considered. Historically, antireflux surgery such Nissen fundoplication has been shown to provide durable relief [5]; however, it is fraught with the risks and invasiveness of surgery and undesirable side effects including dysphagia and gas-bloat symptoms [6,7]. As such, there has been a renewed interest in minimally invasive surgical approaches and endoluminal approaches [8-10]. ransoral incisionless fundoplication using a EsophyX device cannot be performed after 
esophagectomy, surgical sleeve, or on patients with a hiatal hernia greater than $2 \mathrm{~cm}$ [11]. Certain patients who have ongoing GERD symptoms and who have undergone prior Nissen fundoplication will not be good candidates for additional surgical interventions [12]. Additionally, after sleeve gastrectomy, many patients develop significant GERD and are not candidates for either Nissen or transoral incisionless fundoplication. As such, there is a need for a rescue endoscopic procedure for these patients, who otherwise have very few options to manage their GERD symptoms.

The OverStitch endoscopic suturing system (Apollo Endosurgery, Austin, Texas) provides a unique opportunity to provide full thickness sutures over a flexible endoscopic platform [13]. The current version, which received U.S. Food and Drug Administration approval for endoscopic suturing and tissue apposition in 2011, has found use in a variety of settings such as primary endoscopic obesity surgery, post-bariatric surgery endoscopic stoma reduction, perforation closure, covered self-expanding metal stent fixation, gastrointestinal fistula/leak closure, and closure after endoscopic mucosal resection [14].

The aim of this preliminary study was to determine feasibility and safety of endoscopic reinforcement of GEJ using the OverStitch endoscopic suturing system for patients with medically refractory GERD symptoms, including those with altered anatomy from prior surgery or procedures.

\section{Materials and methods}

A total of 10 patients underwent endoscopic reinforcement of GEJ using an endoscopic suturing system (OverStitch) between December 2014 and June 2016. The study was approved by the institutional review board at University of California Irvine (2008-6258). Written informed consent was obtained from all patients. This was a pilot study of consecutive cases. Retrospective analysis of prospectively collected data was done. A validated GERD-Health Related Quality of Life Questionnaire (GERD-HRQL) was administered both before and after the endoscopic procedure to assess changes in symptoms [15]. All patients had symptomatic GERD prior to the procedure. The median duration of GERD symptoms was 5 y (range: 2-10). Five patients had undergone pre-procedural 24-h pH impedance monitoring and all the patients had pathological esophageal acid exposure. All patients with clinical features or diagnostic criteria consistent with GERD, regardless of previous attempted therapies including surgical and endoluminal procedures, were considered for the procedure.

All procedures were performed under general anesthesia in the supine position. Esophagogastroduodenoscopy was performed and markings were placed using an argon plasma coagulator along the 3 o'clock lesser curve from the distal esophagus into the proximal stomach to serve as a landmark to guide endoscopic suturing. Next, suturing was performed using a double channel therapeutic scope affixed with the Apollo OverStitch device. All sutures were placed in a simple interrupted fashion from 12 o'clock to 3 o'clock, followed by 3 o'clock to 6 o'clock, with reinforcement as needed in order to reduce the width of the GEJ ( Figs. 1-5). The GEJ was tightened to the

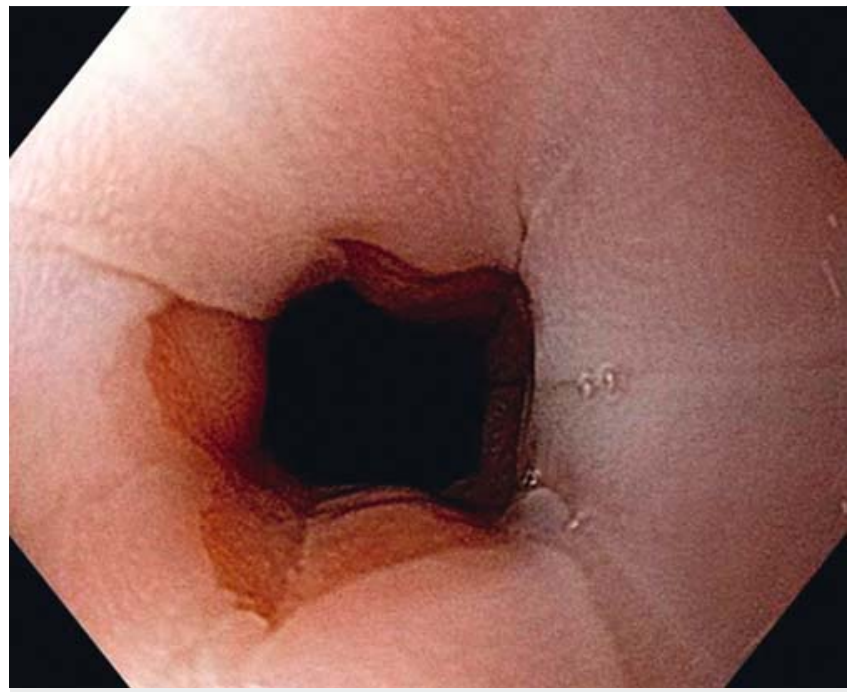

- Fig. 1 Antegrade view of the gastroesophageal junction before endoscopic augmentation.

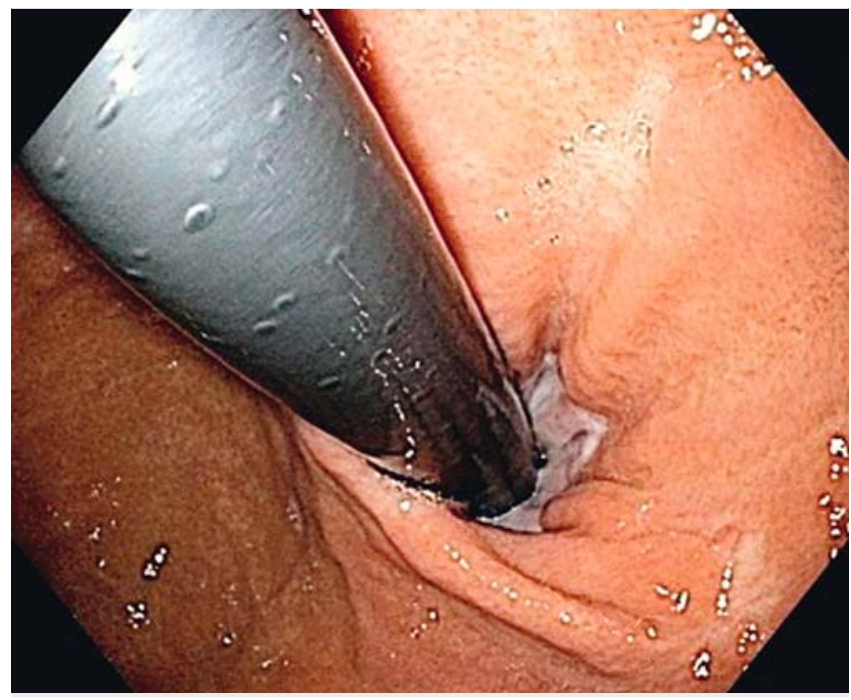

- Fig. 2 Retroflexed view of the cardia before endoscopic augmentation. A diaphragmatic hiatus is noted.

point where the double channel therapeutic scope could be advanced through the GEJ with mild to moderate resistance. Duration of the procedure was defined as time from insertion to withdrawal of the therapeutic scope and Apollo OverStitch system. All patients were administered postoperative pain control and nausea and vomiting prophylaxis and were admitted overnight for observation.

On post-procedure day 1 , routine labs and an upper gastrointestinal swallow using water-soluble iodinated radiopaque contrast medium were obtained to assess for complications such as leak or perforation. Following these evaluations, a diet comprising clear liquids was reintroduced for $24 \mathrm{~h}$, full liquids for $1 \mathrm{wk}$, soft diet for $1 \mathrm{wk}$, and then a regular diet as tolerated. All patients had an initial follow-up visit at $6 \mathrm{wk}$ and additional follow-up visits as determined by the patient and endoscopist. 


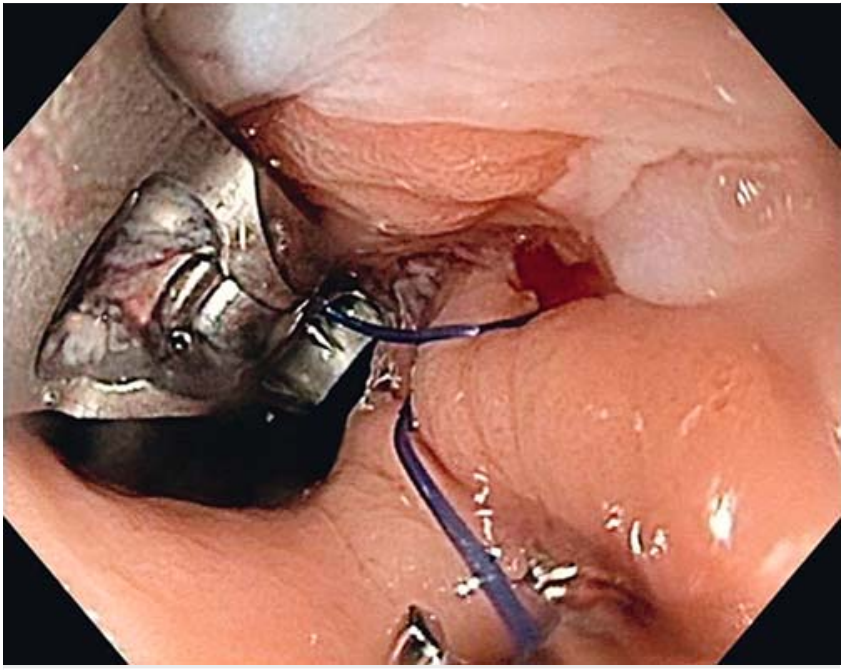

- Fig. 3 A simple interrupted suture was placed from 12 o'clock to 3 o'clock of the gastroesophageal junction using a double channel therapeutic scope affixed with the Apollo OverStitch device. This was followed by another suture from 3 o'clock to 6 o'clock with reinforcement as needed in order to reduce the width of the gastroesophageal junction.

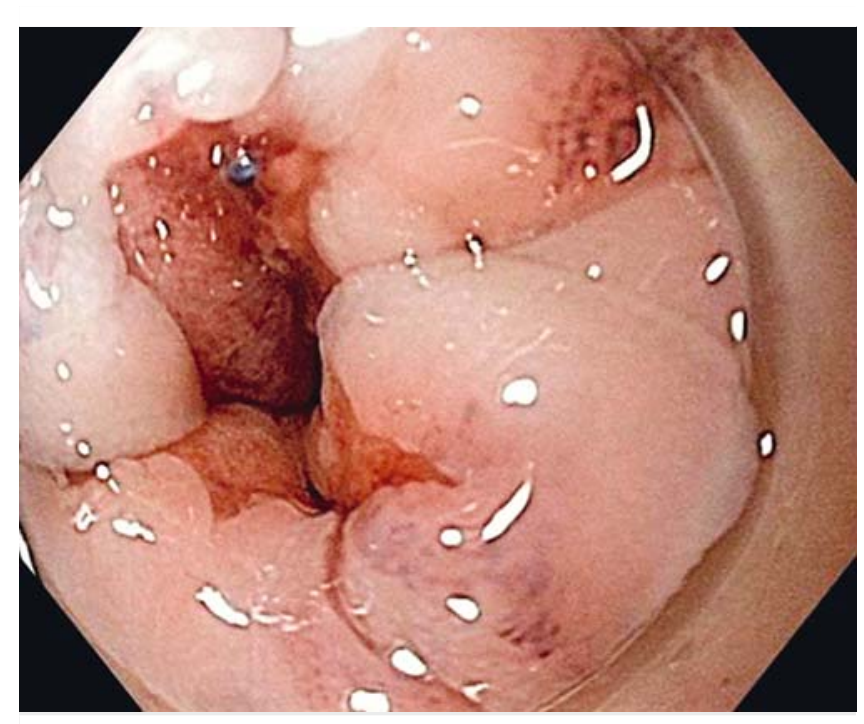

- Fig. 4 Antegrade view of the gastroesophageal junction after completion of the endoscopic augmentation.

At the follow-up visit, the patients filled out the GERD-HRQL. All additional endoscopic procedures, esophageal $\mathrm{pH}$ monitoring, or surgical interventions were recorded.

Statistical analysis was performed using SPSS version 19 (IBM, Armonk, New York). For continuous variables, median and range were calculated. Differences in median pre-procedural and post-procedural GERD-HRQL scores were evaluated using the Wilcoxon signed-rank test.

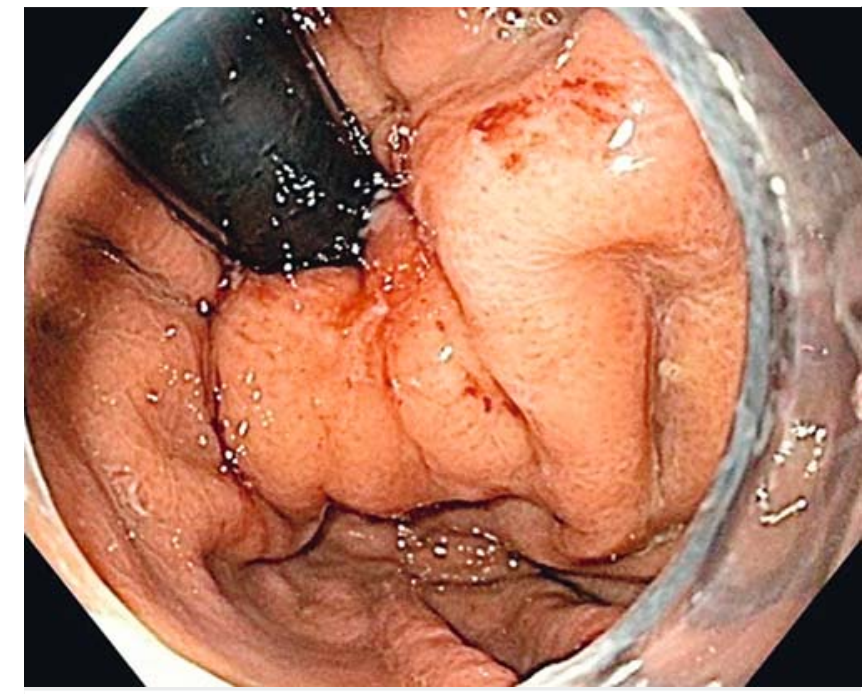

- Fig. 5 Retroflexed view of the cardia after completion of the endoscopic augmentation. There was no hiatus between the gastroscope and the gastroesophageal junction.

\section{Results}

A total of 10 patients with GERD ( 5 female, 5 male) underwent endoscopic reinforcement of GEJ ( $\triangleright$ Table 1$)$. The median age was 61 y (range: $18-75)$. The median body mass index was $25.9 \mathrm{~kg} / \mathrm{m}^{2}$ (range: $21.3-36.6$ ). Seven patients $(70 \%)$ had at least 1 prior antireflux procedure.

Procedure details are shown in $>$ Table 2 . The median number of plications per procedure was 4 (range: $2-8$ ). The median duration of the procedure was 60 min (range: $30-103$ ). A helical retractor device was used in 8 patients (77.8\%). Clinical outcomes are shown in $>$ Table 3 . The median follow-up duration was 5.5 mo (range: 2-12). All patients experienced improvement in their GERD-HRQL scores during the follow-up period. Two patients were able to reduce their PPI usage during the follow-up period. The median pre-procedure GERD-HRQL was 20 (range: $11-45$ ) while the median post-procedure GERD-HRQL was 6 (range: $3-25$ ), a difference that was statistically significant $(P=0.001)$. The median duration of GERD symptom improvement after the procedure was 1 mo (range: $0.5-4$ ). One patient had post-procedural nausea and vomiting that lasted $2 \mathrm{~d}$ and was managed conservatively with anti-emetics.

\section{Discussion}

When established treatments fail to control GERD symptoms, options for less conventional salvage therapies are limited. In this preliminary study, patients with troublesome GERD who had previously been treated with PPIs, endoscopic, or surgical antireflux procedures underwent Apollo OverStitch augmentation of the GEJ with some short-term improvement in their GERD symptoms. A unique benefit of this approach to GERD is the opportunity to customize an endoscopic approach to the patient's anatomy. Technical feasibility was achieved in all patients, including 7 patients with altered anatomy, including 
- Table 1 Patient characteristics.

\begin{tabular}{|c|c|c|c|c|c|c|}
\hline Patient & Age (y) & Gender & Previous treatment & $\begin{array}{l}\text { Hiatal hernia } \\
\text { size }(\mathrm{cm})\end{array}$ & $\begin{array}{l}\text { Hill grade } \\
\text { [19] }\end{array}$ & $\begin{array}{l}\text { Los Angeles grade } \\
\text { esophagitis [20] }\end{array}$ \\
\hline 1 & 64 & Female & Sleeve gastrectomy & 1 & 2 & - \\
\hline 2 & 75 & Male & Nissen fundoplication & 0 & 3 & C \\
\hline 3 & 43 & Male & TIF & 1 & 2 & - \\
\hline 4 & 64 & Female & Radiofrequency augmentation & 1 & 2 & - \\
\hline 5 & 18 & Male & Nissen fundoplication & 5 & 2 & - \\
\hline 6 & 60 & Male & TIF & 0 & 2 & A \\
\hline 7 & 62 & Female & Nissen fundoplication & 1 & 2 & - \\
\hline 8 & 41 & Female & Radiofrequency augmentation & 0 & 2 & - \\
\hline 9 & 39 & Female & None & 1 & 1 & - \\
\hline 10 & 77 & Male & Esophagectomy & 0 & 4 & C \\
\hline
\end{tabular}

- Table 2 Procedure characteristics.

\begin{tabular}{|c|l|l|l|l|}
\hline Patient & $\begin{array}{l}\text { Number } \\
\text { plications }\end{array}$ & $\begin{array}{l}\text { Use of } \\
\text { helical } \\
\text { retractor }\end{array}$ & $\begin{array}{r}\text { Procedure } \\
\text { time (min) }\end{array}$ & $\begin{array}{l}\text { Adverse } \\
\text { events }\end{array}$ \\
\hline 1 & 2 & Yes & 60 & None \\
\hline 2 & 4 & No & 93 & Nausea \\
\hline 3 & 2 & Yes & 60 & None \\
\hline 4 & 4 & Yes & 62 & None \\
\hline 5 & 3 & No & 103 & None \\
\hline 6 & 4 & Yes & 82 & None \\
\hline 7 & 4 & Yes & 60 & None \\
\hline 8 & 4 & Yes & 45 & None \\
\hline 9 & 6 & Yes & 30 & None \\
\hline 10 & 8 & Yes & 58 & None \\
\hline
\end{tabular}

prior fundoplication, esophagectomy, and sleeve gastrectomy. The procedure was also feasible in patients in whom anatomic features such as a large hiatal hernia or diaphragmatic hiatus may preclude other endoluminal approaches to GERD.

The median duration of the procedure was $60 \mathrm{~min}$, which was in keeping with previous studies. Previous studies have shown that the mean duration for peroral endoscopic myotomy mucosal tunnel closure using OverStitch system was $10.1 \mathrm{~min}$ for 1 suture [18]. In our investigational cohort, a median number of 4 sutures (range: 2 - 8 ) was utilized, which would be consistent with comparable procedure duration.

Complications were limited to 1 patient who suffered postprocedural nausea and vomiting, which improved with medical treatment. There were no additional complications such as per- foration, infection, or gastrointestinal bleeding requiring hemostatic maneuvers or transfusion.

There are several limitations in this study. First, this study included a heterogeneous group of patients with GERD. Eight patients had prior surgical or endoscopic treatment of GERD and 1 patient had prior esophagectomy. Six patients had a hiatal hernia, the largest being $5 \mathrm{~cm}$. These confounding factors may hinder evaluation of the efficacy of the procedure. Second, not all patients underwent objective GERD testing with $\mathrm{pH}$ monitoring before and after the procedure, thus relying on the GERD-HRQL questionnaire, which may not be reliable. Third, while the procedure seemed to have improved GERD symptoms initially, at longer follow-up the improvement seemed to disappear. In other words, the effect of the procedure was not durable.

There has been 1 prior study on the use of a full-thickness endoscopic suturing device in GERD. Yanagimoto et al. [16] evaluated the safety, feasibility, and potential effectiveness of endoscopic antireflux funnel creation using a full-thickness endoscopic suturing device in 4 pigs after esophagectomy. The endoscopic antireflux funnel creation was successful in all 4 animals, and the median funnel height was $17 \mathrm{~mm}$. There were no adverse events reported [16]. Since the pigs were euthanized at the end of the study, long-term durability could not be evaluated and further studies are needed to prove efficacy and safety in human patients.

Endoscopic functional luminal imaging probe has been utilized to measure GEJ cross-sectional areas and distensibility in patients with GERD $[17,18]$. Ilczyszyn and Botha evaluated GEJ distensibility before and after Nissen fundoplication in $17 \mathrm{pa}$ tients [17]. Both GEJ cross-sectional area and distensibility decreased significantly after Nissen fundoplication [17]. Rinsma et al. [18] showed that the distending pressure was significantly higher after transoral incisionless fundoplication in 15 patients while the hiatal cross-sectional area did not change. Therefore, GEJ distensibility was significantly reduced after the 
- Table 3 Results of endoscopic augmentation of gastroesophageal junction.

\begin{tabular}{|c|l|l|l|l|}
\hline Patient & $\begin{array}{l}\text { Pre-procedure } \\
\text { GERD-HRQL }\end{array}$ & $\begin{array}{l}\text { Pre-procedure } \\
\text { PPI use }\end{array}$ & $\begin{array}{l}\text { Post-procedure } \\
\text { GERD-HRQL }\end{array}$ \\
\hline 1 & 11 & Daily & 6 & $\begin{array}{l}\text { Follow-up } \\
\text { PPI use }\end{array}$ \\
\hline 2 & 22 & Twice daily & 10 & Twice daily \\
\hline 3 & 28 & Daily & 12 & Twice daily \\
\hline 4 & 13 & Twice daily & 6 & None \\
\hline 5 & 21 & Twice daily & 5 & Twice daily \\
\hline 6 & 11 & None & 3 & Twice daily \\
\hline 7 & 12 & Twice daily & 6 & None \\
\hline 8 & 19 & Daily & 3 & Twice daily \\
\hline 9 & 45 & Twice daily & 25 & Daily \\
\hline 10 & 38 & Twice daily & 22 & Daily \\
\hline & & & & 5 \\
\hline
\end{tabular}

procedure. An endoscopic functional luminal imaging probe may be useful for the calibration of the procedure. In the current study, an endoscopic functional luminal imaging probe was not utilized.

Currently available endoscopic and surgical treatments for GERD cannot be offered to certain patients with prior esophagectomy, sleeve gastrectomy, or prior fundoplication. Endoscopic reinforcement of the GEJ using an endoscopic suturing device does temporarily improve symptoms of GERD, including patients with altered anatomy who would otherwise not have any endoscopic or surgical options available. While the current procedure does show feasibility and safety, a refined technique will be required in order to improve durability to achieve better long-term control of GERD symptoms. Further clinical trials, including those with routine pre- and post-procedure ambulatory $\mathrm{pH}$ testing, in a larger number of patients are needed to evaluate the optimal technique and long-term outcomes of this novel procedure.

\section{Competing interests}

None

\section{References}

[1] El-Serag H, Sweet S, Winchester C et al. Update on the epidemiology of gastro-oesophageal reflux disease: a systematic review. Gut 2014; 63: $871-880$

[2] Peery A, Dellon E, Lund J et al. Burden of gastrointestinal disease in the United States: 2012 update. Gastroenterology 2012; 143: 11791187

[3] El-Serag H, Becher A, Jones R. Systematic review: persistent reflux symptoms on proton pump inhibitor therapy in primary care and community studies. Aliment Pharmacol Ther 2010; 32: 720 - 737
[4] Freedberg D, Kim L, Yang YX. The risks and benefits of long-term use of proton pump inhibitors: expert review and best practice advice from the American Gastroenterological Association. Gastroenterology 2017; 152: $706-715$

[5] Cookson R, Flood C, Koo B et al. Short-term cost effectiveness and long-term cost analysis comparing laparoscopic Nissen fundoplication with proton-pump inhibitor maintenance for gastro-oesophageal reflux disease. Br J Surg 2005; 92: 700 - 706

[6] Wills VL, Hunt DR. Dysphagia after antireflux surgery. Br J Surg 2001; 88: $486-499$

[7] Lundell L. Complications after anti-reflux surgery. Best Pract Res Clin Gastroenterol 2004; 18: 935-945

[8] Garg SK, Gurusamy KS. Laparoscopic fundoplication surgery versus medical management for gastro-oesophageal reflux disease (GORD) in adults. Cochrane Database Syst Rev 2015; 11: CD003243

[9] Ganz R, Edmundowicz S, Taiganides P et al. Long-term outcomes of patients receiving a magnetic sphincter augmentation device for gastroesophageal reflux. Clin Gastroenterol Hepatol 2016; 14: 671 677

[10] Jain D, Singhal S. Transoral incisionless fundoplication for refractory gastroesophageal reflux disease: where do we stand? Clin Endosc 2016; 49: 147 - 156

[11] Håkansson B, Montgomery M, Cadiere GB et al. Randomised clinical trial: transoral incisionless fundoplication vs. sham intervention to control chronic GERD. Aliment Pharmacol Ther 2015; 42: 1261 - 1270

[12] Patti M, Allaix M, Fisichella PM. Analysis of the causes of failed antireflux surgery and the principles of treatment: a review. JAMA Surg 2015; 150: 585-590

[13] Banerjee S, Barth B, Bhat Y et al. Endoscopic closure devices. Gastrointest Endosc 2012; 76: $244-251$

[14] Stavropoulos S, Modayil R, Friedel D. Current applications of endoscopic suturing. World J Gastrointest Endosc 2015; 7: 777-789

[15] Velanovich V. Comparison of generic (SF-36) vs. disease-specific (GERD-HRQL) quality-of-life scales for gastroesophageal reflux disease. J Gastrointest Surg 1998; 2: 141 - 145

[16] Yanagimoto Y, Yamasaki M, Nagase $\mathrm{H}$ et al. Endoscopic anti-reflux valve for post-esophagectomy reflux: an animal study. Endoscopy 2016; 48: 1119-1124

[17] Ilczyszyn A, Botha AJ. Feasibility of esophagogastric junction distensibility measurement during Nissen fundoplication. Dis Esophagus 2014; 27: 637-644 
[18] Rinsma N, Smeets F, Bruls D et al. Effect of transoral incisionless fundoplication on reflux mechanisms. Surg Endosc 2014; 28: 941 - 949

[19] Hill LD, Kozarek RA, Kraemer S] et al. The gastroesophageal flap valve: in vitro and in vivo observations. Gastrointest Endosc 1996; 44: 541 547
[20] Armstrong D, Bennett JR, Blum AL et al. The endoscopic assessment of esophagitis: a progress report on observer agreement. Gastroenterology 1996; 111: 85-92 